\title{
Collaborative Communication Tools for Designing: Physical-Cyber Environments
}

\author{
Stephen Forshaw \\ HighWire \\ The LICA Building \\ Lancaster University \\ Lancaster, Lancashire \\ LA1 4YW \\ United Kingdom \\ s.forshaw1@lancaster.ac.uk
}

\author{
Leon Cruickshank \\ Imagination Lancaster, \\ Lancaster Institute for \\ the Contemporary Arts, \\ Lancaster University, \\ Lancaster LA1 4YW, UK \\ L.Cruickshank@lancaster.ac.uk
}

\author{
Alan Dix, \\ Talis, Birmingham, UK \\ and School of Computer Science, \\ University of Birmingham, UK. \\ alandix.com
}

\begin{abstract}
Designing for Physical-Cyber Environments (P-C E) will require a collaborative interdisciplinary approach. A Physical-Cyber Environment is my interpretation of an emergent hybrid, physical, digital mix, firmly grounded in physicality, which is technologically, digitally enabled and augmented. This landscape (P-C E) is an emerging possibility space where all types of products, services and environment will be possible. Designing for such complex environments will require the involvement of various disciplines, stakeholders and end end-users when appropriate. Each of these bringing with them their own internalized assumptions and thought processes, making understanding and discussion between the various parties potentially problematic. Tools are needed to aid productive dialogue between those involved. In this paper a selection of technologies in varying stages of development and concepts from science fiction are introduced to help describe the Physical-Cyber Environment. A discussion regarding difficulties in interdisciplinary collaboration and a description of a workshop called the "Alien Technology Workshop" designed to explore tools to aid productive collaborative discussions is also introduced.
\end{abstract}

Keywords Hybrid, Physical-digital, physicality, design-tools-methods, interdisciplinary collaborative, Alien Technology, communication tools, design process.

\section{INTRODUCTION}

Imagine a future where the physical and digital become seamlessly intertwined producing a strange new hybrid landscape. Where technologies have the potential for virtually unbounded possibilities. In this paper the author introduces the idea of an emergent physical, digital hybrid space called a "Physical-Cyber Environment" (P-C E) and an interdisciplinary workshop called the "Alien Technology Workshop".

This Physical-Cyber environment, although not a reality at present in its full manifestation, is in the context of this paper a possibility spaced and proposed as emerging. Currently things physical and things digital are already converging into hybrid objects and environments and the world of ubiquitous embedded computation continues to grows rapidly. [24][32] The realm of the PhysicalCyber Environments is an umbrella term to help describe a landscape, forming from an aggregation of many technologies, materials, systems and innovations. These may include: ubiquitous computing,[17] Cyber-Physical systems, ambient intelligence [1], physical digital hybrids,[22] smart materials,[6] augmented reality, [2] mixed reality [13] [20], cross reality, [18] and embodied virtuality. [32] Figure 1. depicts physical and digital merging into the hybrid Physical-Cyber Environment. Whilst looking at this landscape, a selection of research projects and concepts are presented to help describe the idea of Physical-Cyber Environments. These include: Lightspace, Home of The Future, Mirage and science fiction based concepts.

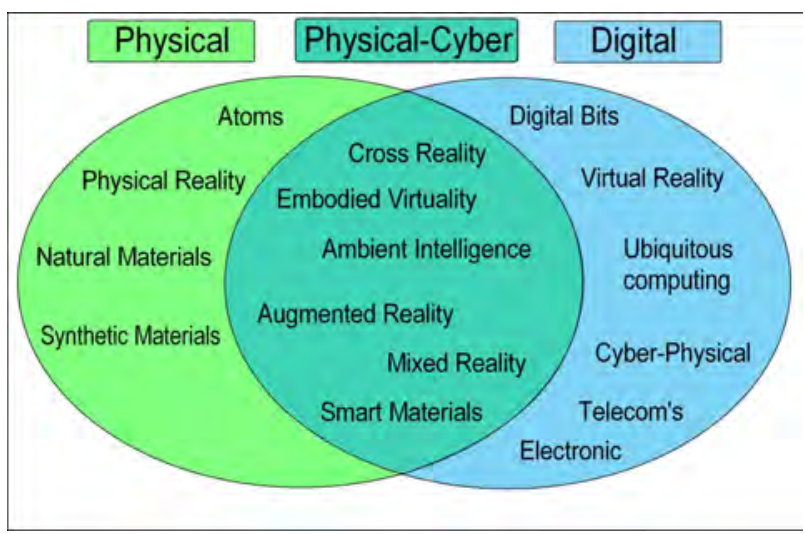

Figure. 1 Emergent Hybrid Space: P-C E.

The concept of the emerging hybrid space (P-C E) builds upon and expands ideas and concepts discussed by other researchers into hybrid spaces. For example the latest Blast Theory game "I'd Hide You" [3] which combines the physical reality of an urban space ( Manchester city centre) where 
'performers' wearing cameras are tracked and interacted with via GPS and live video streaming by online game players, mixing virtual and physical elements. Steve Benford and Gabriella Giannachi [12] collaborative project 'Day of The Figurines' which likewise mixed the physical elements with virtual element to create a gaming experience. In this game the players can interact via text messaging with a game operator to effect and move physical game figurines from one position to another on the game board. Players can also interact with one another electronically to share knowledge and objects within the game.

Adriana de Souza e Silva in her article 'From Cyber to Hybrid' [8] discusses the blurring of borders between physical and digital spaces via the mobility of users and their mobile devises. She explains her definition of a hybrid space as one that occurs "when one no longer needs to go out of physical space to get in touch with digital environments".

Paul Dourish discusses [10] 'place and space' in light of mobile technology and how one is affected by the other. Having defined space as that which is the geometric configuration and place being that of the the social meaning and 'understood reality' he explains how the introduction of 'locative media' via technology into a space can change its meaning. He argues that the overlaying of "real" spaces with "virtual spaces" in a physical world embedded with technology provides new meaning and new ways in which that world is understood. He puts forward a different interpretation of space and place where the physical world is not separate from the technologically mediated world from which "new cultural practices" and "new forms of environmental knowing" emerge.

These interpretations and discussions of hybrid spaces, helps to describe the genesis of hybrid spaces. However for the purposes of this paper the Physical-Cyber Environment hybrid space is that of an evolution into a broader perspective of the hybridisation of the physical world. The term Physical-Cyber Environment describes the gradual aggregation of many different technologies and systems including digital mixed with the physical.

It is this author's contention that designing for such complex hybrid landscapes will require interdisciplinary collaboration, which may include stakeholders and end users participation. It is recognised that currently the design of complex systems often involve interdisciplinary collaboration and many design houses utilise participatory design methods. These strategies can be problematic concerning issues regarding, time, cost and communication difficulties encountered in involving other disciplines, stakeholders and end users [26]. However in this paper the author is concerned with one problematic aspect of those strategies, that being interdisciplinary communication difficulties. This paper introduces the 'Alien Technology Workshop' set within an imaginary scenario, where tools to aid facilitation of productive interdisciplinary discussions are explored. This particular technique "Alien Technology" aims to help participants be more at ease and open to collaborative discussion, where participants are equally inexpert and are encouraged to postpone judgement. The aim is to aid participants in externalising, recognising and valuing differences in disciplinary cultures.

The folowing sections contains a selection of technologies which help describe Physical-Cyber environments, followed by a discussion of interdisciplinary collaboration in design, then followed by a description of the Alien Technology workshop.

\section{TECHNOLOGIES \\ 2.1 Lightspace}

Lightspace [19] is a project which draws together aspects of augmented reality and surface computing producing in combination a deeply interactive environment. The system enables the environment so that any physical surface can become interactive and also the actual space between surfaces all become 'fully interactive'. The Lightspace system is a combination of different technological artefacts including several depth cameras and video projectors to produce an interactive spatial computing environment. Within this environment it is possible to move data, represented visually, from one place to another, for example from a wall to a table. The system also facilitates the shifting of data from one person to another as they interact with projected images passing them from one person to another. The Lightspace environment is calibrated so that projected elements are mapped to real world coordinates. This in turn means that any surface set within the Lightspace environment can potentially become an interactive artefact, including the open space. People are able to interact with the environment by way of gestures and multi-touch interactions with surfaces. In one example a person was able to hold out their hand (palm facing up) into a projected beam, which in-turn acted as a sort of menu, at which point raising or lowering the hand induced different options to appear in the palm of the hand.

\subsection{Ambient Intelligence / intelligent environments}

The UK's Channel 4 documentary series "Home of the Future" [7] explored a variety of technologies which created an intelligent environment for the inhabitants. The house had been fitted with new 
and experimental technologies to investigate how the family living there would respond to such an environment. This is a form of possible end user showroom / prototype testing. [14] Some of the technologies included: Eco-power systems to help control power consumption and explore new domestic power systems, various sensors to tailor temperature and lighting to the individuals, entertainment and leisure systems, Smart materials for example clothing which responded to music, an intelligent bathroom mirror connected with health and fitness and devises which monitored brain activity. In this experiment the family on the whole were positive and receptive to the technologies they had to live with. However some difficulties were experienced for example: personalised automated temperature regulation of bath water, appears to have not always delivered desired results which affected user confidence in the technology. Another example was end user habits in contrast to automated systems, in one case the father habitually switched power off to save electricity, which clashed with the automated systems needed to regulate power usage.

\subsection{Mirage}

Mirage [31] is being developed at the Virtual Reality Applications Centre, lowa State University, this is a three dimensional, fully immersive, synthetic environment. This environment contains back projected images on the walls ceiling and floor, eight channel surround sound, haptic force feedback, physical objects, virtual and augmented reality and tracking systems. Mirage and the various other projects in development at the centre are interdisciplinary collaborations between the research lab, government and industry. The centre is researching and developing a variety of these environments aimed at leisure, education, business and military uses.

\subsection{Science Fiction}

Increasingly science fiction presents ideas of interactive, immersive, augmented environments. Notably is the fictitious Holodeck set within the Starship Enterprise featured in the Next Generation Star Trek series. This example pushes the imaginary boundaries to the limits as the Holodeck is not only able to produce a visual environment but also touchable physical interactive objects of just about any kind, which can be manipulated, felt and even sat on. It can even simulate corporeal entities such as people who are fully interactive.

Nevertheless a holodeck environment, like the one featured in Star Trek appears to be a sort of holy grail for some in pursuit of augmented reality.

Most recently the film Prometheus, the 2012 prequel to the Alien films depicted a spatially interactive navigation system which surrounded the pilot. Not only did this system appear as visual augmented reality but the planets could be manipulated physically, a sort of hybrid tangible user interface. Nathan Shedroff and Chris Noessel [27] offer the suggestion that lessons can be learned from science fiction interfaces in the development of real world interfaces. They speak of a two way influence on design, one being real world design influencing hybrid science fiction interfaces. The other being science fiction influencing real world interface design by inspiration, expectation, social context and the innovation of new paradigms. For example the Motorola Star-TAC flip phone bears similarity to the flip communicator from the Star Trek original television series. Lab research of gesture interfaces like g-speak platform of oblong industries inspiring gestural interfaces of films like The Minority Report.

These examples demonstrate that the designing of complex hybrid environments involve the collaboration and expertise of various disciplines and stakeholders. Collaboration can however bring with it some communication challenges between those parties involved where difficulties making their ideas explicit can arise. [21]

To explore some of these challenges a discussion about interdisciplinary communication difficulties is put forward, a workshop technique "Alien Technology" and a collaborative "Communication Tool-kit" is introduced as an aid to encourage productive communication.

\section{INTERDISCIPLINARY COLLABORATION IN DESIGN}

To produce valued solutions in design, there is a need for frameworks and tools which take into consideration and can help to bring together multidisciplinary groups from within specialized areas of expertise.[15] Cross discipline collaboration can improve the possibility of innovative and effective solutions. [28] A growing challenge to design practice is the need to bridge the communication gap between various professions, designers, other stakeholders and end user groups involved in the design process.[26] Even within sub departments of organisations people have "unique perspectives"of aims and tasks causing conflict. The "sharing of perspectives" is seen as helpful in this conflict and that becoming aware of conflicting ideas and discussions about them can be useful. [4] Ethnography suggests that collaboration can be enabled by shared representation, these externalized representations add to cognitive processing. Externalization of individuals thoughts and ideas via representation in artefacts can aid communication of those thoughts and ideas. [9] 
Myra Strober's studies into interdisciplinary conversations [28] have shown some of the difficulties faced by interdisciplinary teams which include differences in: language, ways of thinking, assumptions, ideas, ways of presenting, discerning and evaluating. These are described by Myra as "discipline cultures" and "habits of mind". Myra Strober's study over several years makes some suggestions concerning the barriers between the disciplines and strategies which could help to make the conversations more productive. These suggestions are: Start by introducing the idea of disciplinary cultures to tightly structure sessions, making apparent the purpose of the session, selecting participants who are interested in syntheses between disciplines, establishing trust between the participants by being selective of participants with good interpersonal skills and distribution of conversation among the participants avoiding monopolization, introduce the participants to the idea of differences in disciplinary "cultures", agreement on some base rules for the session by the participants. Liora Salter and Alison Hearn in their book on issues in interdisciplinary research [25] they note that it took two years to be effectively submerged in a new culture. Myra Strober similarly emplanes that at Bio $X$ an interdisciplinary science centre at Stanford, it took two years of weekly meetings to learn the culture and habits of mind of each others disciplines.

Such a time-scale poses a problem for interdisciplinary / collaborative design teams as they may not have the luxury of two years to learn multiple discipline cultures and habits of mind. Therefore time effective solutions need to be explored to begin to address some aspects of developing productive communication between various and diverse collaborators. Some of these issues and suggestion are explored in the context of the Alien Technology workshops. The workshops overall aims are to help collaborators recognise and value differences equally in disciplinary cultures through the externalisation of differences.

\section{THE ALIEN TECHNOLOGY WORKSHOPS}

The Alien Technology workshops are a series of workshops investigating the development of a toolkit to aid productive interdisciplinary communications in a collaborative design process set within a landscape of Physical-Cyber

Environments. Each workshop focuses on different elements in the development of productive communication. The first workshop being a pilot study exploring the externalisation of the participants design process, as process is mostly hidden within a designers mind. [16] The second workshop (ATW 1.0) investigating the externalisation of disciplinary differences in assumptions, interpretations, representations and modes of presentation. A third workshop (ATW 2.0) exploring the recognition, valuing and synthesis of ideas. A fourth workshop (ATW 3.0) exploring collaborative design for the application of technology. The Alien Technology technique is used as a method to explore the design and development of such emergent hybrid spaces. The Alien Technology technique includes a fictitious scenario including roles, props and objectives. The scenario includes the participants taking on the role of an alien with access to advanced technology, this technology is called "Alien Putty". While in this role they are expected to consider an alternative alien culture and their environment which they have limited information about and at some stage they consider the application of "Alien Putty" to that environment. Giving participants a scenario provides context to the task in which to frame ideas and thinking about an unknown environment, technology and end user groups. The fictitious nature of the scenario enables some ambiguity [11] and freedom of imagination on the part of the participants in the design process. In this scenario there are no experts within the group and therefore the participants are equally inexpert. All participants should be viewed as equally valuable in the creative process. There is no right or wrong, all participants can create and contribute in a safe non threatening environment. A role to play in the scenario helps to immerse the participant into the scenario enabling them to engage and participate in the task given. In this scenario the role is fictitious to add a fun element and put participants at ease allowing them to be more open towards collaborative discussions.

The alien environment represents a Physical-Cyber Environment. The participant aliens represent a collaboration of disciplines and stakeholders. The alternative alien culture represent the end users of an environment. The Alien Putty represents technology with virtually no constraints and seemingly limitless uses. It is a metaphor for known and unknown abilities and possibilities that technologies may bring in the future. The 'Alien Putty' it is used in a conceptually similar way to 'dream tools' [5]

\subsection{Alien Technology Pilot Study}

A pilot study was undertaken to explore the concept of the Alien Technology scenario and the externalisation of the participants design process, as process is mostly hidden within a designers mind. [16] The pilot study focused on individuals from different disciplines. The participants were given the role of being an alien and a scenario where their mission was to plan, how they might undertake the application of their own "Alien Technology" to enhance another alien culture's environment. 


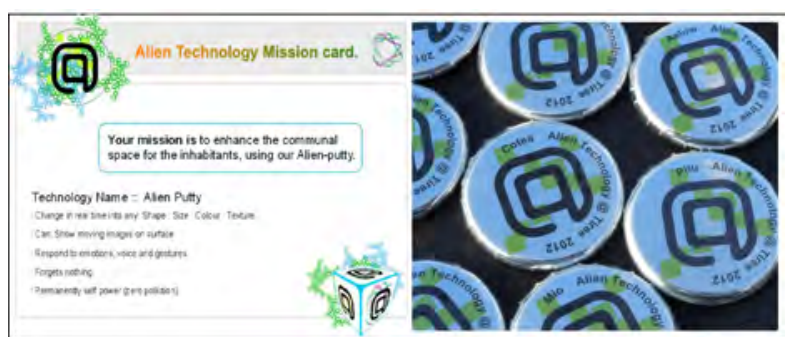

Figure 2 Pilot Study Mission Card and Badges

A prop was introduced to them representing the alien technology, in the form of "Alien Putty" figure 3. and is represented by soft modelling clay. In the context of the workshop the Alien Putty is a technology which can do virtually anything the participants can imagine. This provided a physical focal point to interact with which aided them in expressing their process, thoughts and ideas verbally and on paper. The participants were asked to use verbal protocols to externalise their thinking.

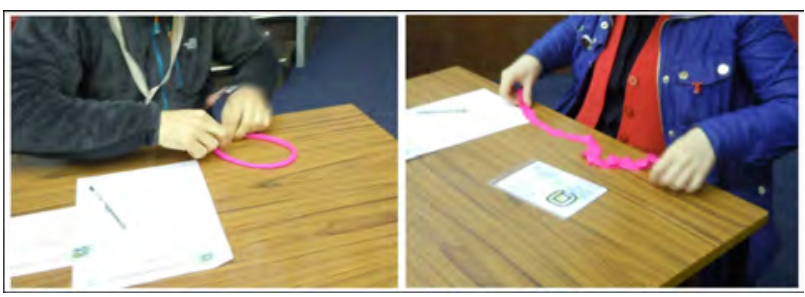

Figure 3. Interacting with the "Alien Putty"

The participants of the pilot study were drawn from a mixture of artists, software engineers, $\mathrm{HCl}$, product designers and business management. In this mix were Practitioners, Professors and Ph.D students, see table 1.

Table 1. Pilot Study Participants

\begin{tabular}{|c|c|c|c|}
\hline $\begin{array}{l}\text { Participant } \\
\text { Code }\end{array}$ & Date & Profession /Discipline & Location \\
\hline A1 & $15 / 3 / 2012$ & Artist & LICA \\
\hline A2 & $15 / 3 / 2012$ & Software Engineer & LICA \\
\hline A3 & $15 / 3 / 2012$ & Business & LICA \\
\hline A4 & $15 / 3 / 2012$ & Software Engineer & LICA \\
\hline A5 & $15 / 3 / 2012$ & Product Designer & LICA \\
\hline A6 & $23 / 3 / 2012$ & Software Engineer & TTW3 \\
\hline A7 & $23 / 3 / 2012$ & Product Designer $\mathrm{HCl}$ & TTW3 \\
\hline A8 & $24 / 3 / 2012$ & Artist & TTW3 \\
\hline A9 & $24 / 3 / 2012$ & Software Engineer & TTW3 \\
\hline A10 & $24 / 3 / 2012$ & Software $/ \mathrm{HCl}$ & TTW3 \\
\hline A11 & $24 / 3 / 2012$ & Software Engineer & TTW3 \\
\hline
\end{tabular}

Findings showed that the alien technology scenario and roles where adopted readily by the participants and they were at ease with their roles. This workshop demonstrated that the introduction of the fictitious alien scenario gave participants a context on which they were able to comment and verbally externalise their process.

Additional findings of the study emphasized some differences in the assumptions made by professionals from different disciplines. It was also observed that the participants tended towards two distinct approaches to their process with regards to consideration of the end users. These were, those who would take an ethnographic approach and those who would build it and see what happens approach. During the session there was opportunity for the participants to make notes, some of the participants did make notes whilst other preferred not to, demonstrating some differences in preferred modes of communication. The aims and findings of the pilot study were successful in helping to frame the first Alien Technology workshop 1.0.

\subsection{Alien Technology Workshop 1.0}

The aim of the Alien Technology workshop 1.0, was to investigate tools to aid the externalization of disciplinary assumptions and differences between participants in an interdisciplinary team within a landscape of seemingly unbounded technology.

The workshop attempts to do this in a number of ways:The introduction of the Alien Technology scenario. To introduce the idea of differences in disciplinary assumptions, thought processes and modes of representation and presentation. The introduction of a communication kit figure 6 . to aid productive communication. In the context of these workshops the communication tool kit is used in a face to face setting and at this stage virtual participants are currently not involved. ATW 1.0 had a duration of one hour. The elements of the workshop are as follows:

\section{Warm-up}

A warm up activity to externalise some differences in assumptions based on the phrase 'space alien' where the participants create their own model of a space alien and give it a name. This helps participant 'postpone judgement' and give permission to push the boundaries to the nonsensical in a non threatening way. [29] Each participant then briefly introduces themselves and the space alien they have made to their group.

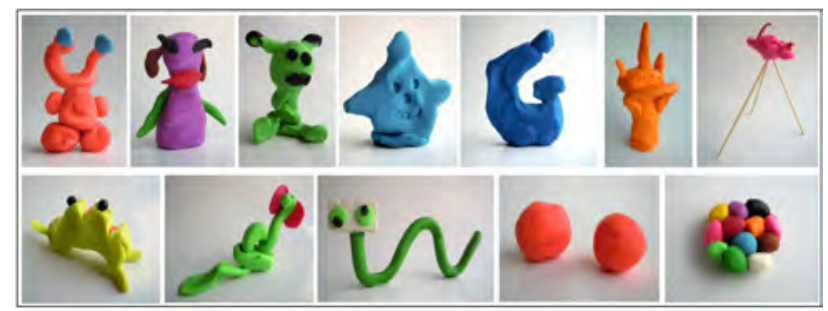

Figure 4. Space Aliens

Main Task

The introduction of vignettes, artefacts that aims to ensure all the participants are responding to the same materials, these also act as controls for the research. In this workshop the artefacts are reconnaissance pictures figure 5 . from the alien environment, which the participants are encouraged to interpret and then are asked to 
present their interpretations to the group. This task reinforces some of the differences in thoughts and ideas of the participants. The reconnaissance images are purposely ambiguous however suggest some form of intelligently constructed environment.

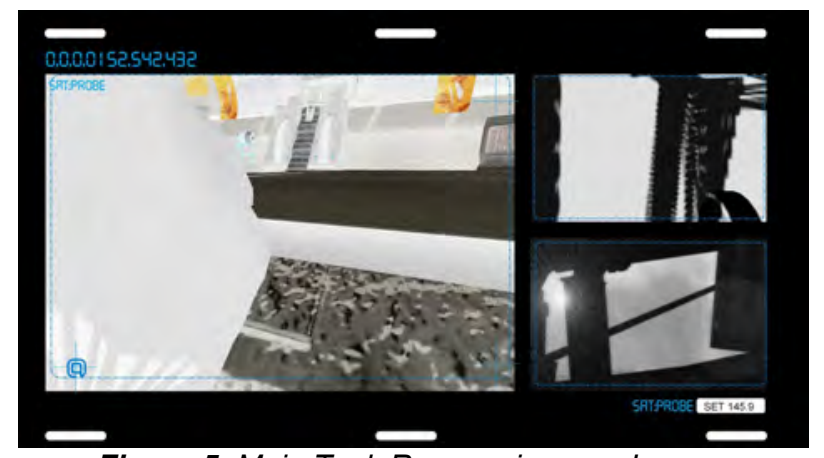

Figure 5. Main Task Reconnaissance Images

A communication kit figure 6 . containing a considered selection of materials to aid articulation of ideas and thinking between the different disciplines. These materials are intended to allow for different modes and styles of representation of ideas, thinking and presentation, making them accessible to different participants preferences and require no specialised skills to use. [23] The communication kit comprises of $3 D$ and 2D materials and a variety fixing / fastening and markmaking items. The 3D items included: Lego, K'nex, Construction Shapes and Modelling Clay. The 2D items included: Sticky-notes, various sized notepads, a selection of A4 paper / card and an A2 sketch / flip pad. These resources are included to facilitate low-fidelity 3D models, sketches and notation. The participants make their own selection of materials from the communication kit to create their individual interpretation and representation of the environment. The participants were instructed to use the items in any way they chose to represent their individual interpretations.

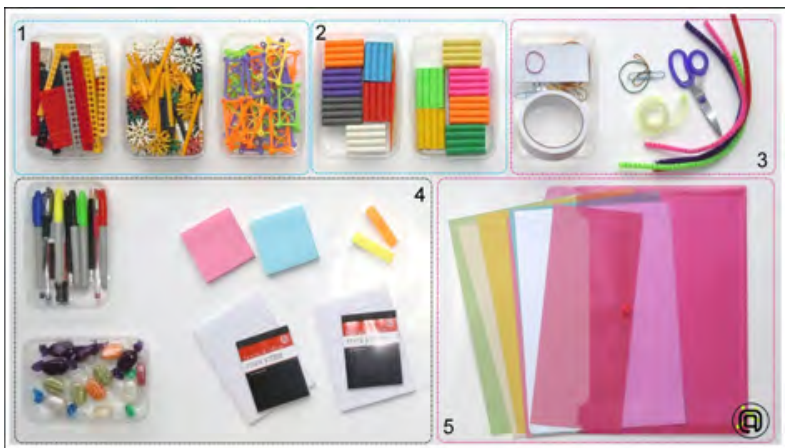

Figure 6. Communication Kit

Presentations

Presentations, the participants present their individual representations, interpretations, ideas figure 7. and key characteristics to their group, allowing other participants to view and discuss the different interpretations and modes of representation and presentations.

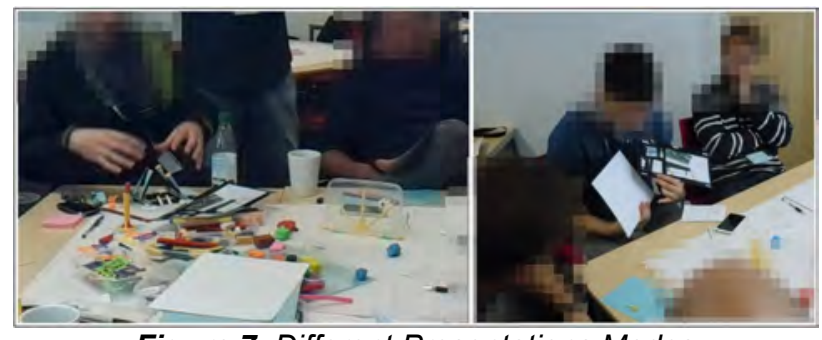

Figure 7. Different Presentations Modes

The participants are reunited with their space aliens (made earlier) and then asked to introduce their space alien to their environment figure 8 . This gives the opportunity for participants to interact with their space alien and environment as a fun element if they chose to. This also serves to link the space alien with its name tag to the interpretation work on the table for reference.

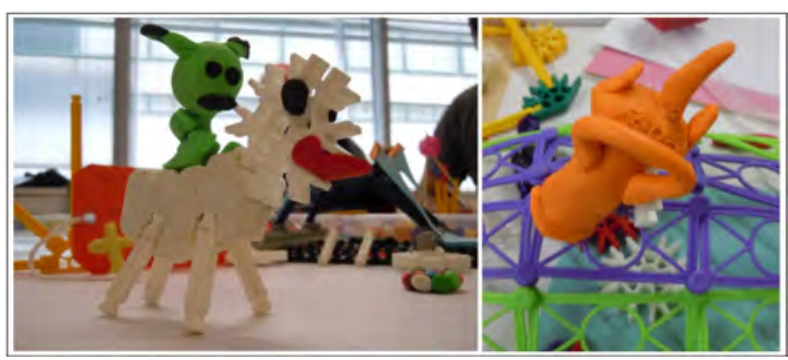

Figure 8. Reunited Aliens

The facilitator rounds up by emphasising some of the benefits of externalising assumptions and differences in disciplinary cultures, in aiding productive communication between disciplines. It is hoped that the participants can take away with them the idea of looking for and recognising disciplinary differences with a view to valuing those differences equally in a interdisciplinary collaborative setting.

The participants of workshop 1.0 were drawn from a mixture of artists, computer sciences, business management and organisational science. In this mix were Practitioners, Professors and Ph.D. students, see table $2 \& 3$.

Table 2. Group B: Presentation Modes

\begin{tabular}{|cll|}
\hline $\begin{array}{c}\text { Participant } \\
\text { Code }\end{array}$ & Group B, Mode of Interpretation / Presentation & Profession / Discipline \\
\hline B1 & Clay, K'nex, Other \& Smart-Phone & Project Management \\
\hline B2 & Notes, Drawing on card \& Smart-Phone & Management \\
\hline B3 & Notes & Management \\
\hline B4 & Notes + drawing, Lego \& Smart-Phone & Computing Science \\
\hline B5 & Clay, K'nex, Paper & Management \\
\hline B6 & Clay, K'nex, Lego, Paper \& Smart-Phone & Computing Science \\
\hline B7 & Notes with red and blue ink \& Smart-Phone & Software / Art \\
\hline
\end{tabular}


Table 3. Group C: Presentation Modes

$\begin{array}{cll}\begin{array}{c}\text { Participant } \\ \text { Code }\end{array} & \text { Group C, Mode of Interpretation / Presentation } & \text { Profession / Discipline } \\ \text { C1 } & \text { Clay, K'nex, } & \text { Business } \\ \text { C2 } & \text { Clay, K'nex, Lego, Pipe Cleaners, Shapes,Notes, } & \text { Management } \\ \text { C3 } & \text { Other } & \\ \text { Clay, Card, Pipe Cleaner, Pencil markings,Notes } & \mathrm{HCl} / \text { Interact Art } \\ \text { C4 } & \text { Clay, K'nex, Shapes, } & \text { Computing Science } \\ \text { C5 } & \text { Clay, K'nex, Lego, Pipe Cleaners, Shapes, } & \text { Organisational Science } \\ & \text { Rubber Bands, Other } & \text { Computing Science } \\ \text { C6 } & \text { Clay, K'nex, Shapes } & \end{array}$

\subsection{Findings}

The overall findings of ATW 1.0 generally supported the aims of the workshop in that the communication kit appeared to aid the externalisation of the participants assumptions, ideas and interpretations. The selection of resources provided in the communication kit did allow for some differences in modes of representation and presentation to be observed and recognised by other group participants. The selection of resources in the communication kit appeared to be accessible to all participants. The participant demonstrated a wide variety of differences in their assumptions and interpretations derived from the phase "space alien". Some created space aliens with humanoid features, some were creature like and others were abstract, figure 5. some gave their space aliens special attributes . Throughout the main task it was observed that all participants externalised their thinking in some way, verbally, visually, through gestures, note form, 2D sketches or 3D models. The individual interpretations of the reconnaissance image varied widely creating some discussion within each group see figure 9 .

There were distinct differences between the two groups. In Group B individual and disciplinary differences were easier to observe than in Group C. During the workshop the two groups appeared to approach the main task somewhat differently. Group B appeared to approach the task in a more cautious and systematic manner with a playful aspect. It was observed that Group B began the main task by predominantly spending some time contemplating the reconnaissance images before shifting into the representative phase. Whereas some participants in Group C seemed to approached the task more impulsively seemingly playing rather than being playful. In Group $\mathrm{C}$ most participants appeared to go directly into the representative phase after viewing the image briefly, predominately building and making utilising the communication kit.

Group B carried out their task mostly in silence in an insular manner and appeared very focussed on producing their own interpretations. Group $C$ carried out their task in a seemingly more sociable manner. In group $C$ there was more discussion during the task, some social and some related to the task, discussing ideas about the interpretations.
The representations produced by Group B were more varied in mode than those of Group C. Group $B$ members appeared to adhere to their own discipline modes of representation, some externalised their thinking in note form figure 9.4, 9.9 some through sketches, figure 9.6 others through model making figure 9.3. In Group C most members used similar modes to one another in their representations, externalised through model making, figures 9.1, 9.2, 9.7. However in Group C there appeared to be some recognisable disciplinary influences in their interpretations, use of materials and attitude towards the materials provided. In Group C one member used card figure 9.8 to form and build their representation whilst the rest of Group C used the preformed materials such as Lego. In Group B paper and card where used as predominantly as materials for writing and drawing on figure 9.5. A few members of Group C seemed to view some of the materials provided as merely play things.

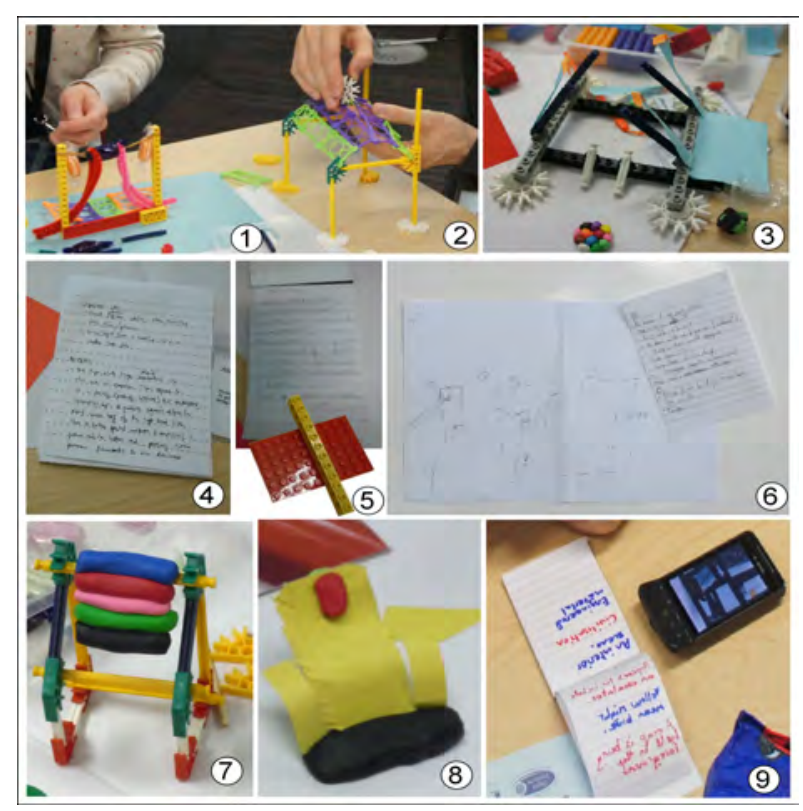

Figure 9. Different Interpretation Representations

During presentations the participants demonstrated a variety of differing modes. One stood and read from notes some used the reconnaissance image as a visual aid. Some used their models as aids, others used a combination of the reconnaissance image and their models / sketches as visual aids figure 7 .

Following the individual presentations, some of the participants held a discussion concerning differences they had noticed during the tasks.

Others commented that they would have liked more time to reflect and discuss disciplinary differences. Some participants expressed how they would have liked to continue on into a group phase to synthesise their ideas about their interpretations of the environment. One participant commented that 
the workshop had been "a revelation" regarding observation of process.

Further workshops are planned (WT 2.0 \& WT 3.0) to continue this line of inquiry, where the collaborative design elements of the workshop will be introduced while implementing the communication kit to aid productive dialogue, for the exploration of creative interdisciplinary collaboration within a landscape of seemingly unbounded technology. Further workshops are currently in the design and development stage.

\section{CONCLUSIONS}

In the future design of Physical-Cyber Environments (P-C Es), where the physical and digital become seamlessly intertwined, incorporating technologies of seemingly unbounded possibilities an interdisciplinary collaboration of professionals, stakeholders and end users (when appropriate) will be needed. This paper has put forward some of the difficulties faced by interdisciplinary collaborative teams and some of the suggestions made by others who have researched into this area and a workshop based communication kit to aid productive communications. It is recognised that there is no one panacea to address these difficulties and that more tools are needed to aid these interdisciplinary discussions in the design process. The workshop tools put forward in this research, although in the early stages, preliminary findings have demonstrated that externalisation of differences in assumptions, modes of representation and presentation can be helpful in developing some recognition of these differences which may lead to valuing equally differences in disciplinary cultures. This recognition and valuing of disciplinary cultural differences are necessary steps towards productive communication in interdisciplinary groups. The communication kit used in the Alien Technology workshop contains a variety of materials and artefacts to encourage externalisation of ideas and thoughts which consider differing modes of communication. This communication kit could be a useful resource for design teams in the early stages of a interdisciplinary collaborative project, where externalisation of assumptions, thoughts, ideas, differing representation and presentation modes could be beneficial in aiding productive dialogue.

The Alien Technology technique used in the workshops essentially comprises of a series of stages, incorporating the interdisciplinary communication kit and a fictitious scenario as part of a design process. The stages form a framework to aid productive communications alongside the communication kit. The stages are as follows: 1.Externalising assumptions about the end user. 2.Externalising differences in ideas, thinking, modes of representation and presentation.
3. Reflection and discussion, to aid recognition and valuing of differences.

4.Synthesis of ideas.

5.Ideation and application of technology.

The "Alien Technology workshop" using the Alien Technology technique is seen as a workshop that could be implemented at the start or early stages of a real world interdisciplinary project, or as an introduction to disciplinary cultures in business and academia seeking interdisciplinary approaches during the development of design ideas for emergent hybrid technologies and environments (P-C Es).

The Alien Technology technique incorporating a real world scenario could also provide a useful framework to follow as a method to continue productive communications throughout the design process of a real world project.

\section{REFERENCES}

[1] Aarts, E.; Philips Res., (2010). Ambient intelligence: a multimedia perspective. Multimedia IEEE: Jan. March 2004 - Volume: 11- Issue:1- pp $12-19$

[2] Benko, H. Ishak, E W. Feiner, S. (2005) CrossDimentional Gestural Interaction Techniques For Hybrid Immersive Environments. Proceedings of the IEEE Virtual Reality 2005, VR 05. Germany, March $12^{\text {th }}-16^{\text {th }} 2005$, pp 209-327.

[3] Blast Theory (2012) l'd Hid You [Available Online] Future Everything 2012, 17-19 May http://www.idhideyou.com/

[4] Boland, R. Schwartz, D. Tenkasi, R. (1992) Sharing Perspectives in Distributed Decision Making Proceedings of CSCW 1992, November pp306 - 313

[5] Brandt, E. Grunnet, C. (2000) Evoking the future Drama and props in user centred design Proceedings of Participatory Design Conference 2000

[6] British Parliament (2008). Smart Materials. [Online] The Parliament office of science and technology. Available from:http://www.parliament.uk/documents/post/post pn299.pdf. [Accessed 6/6/2011]

[7] Channel 4 Home of The Future First Broadcast 7 pm Sunday $12^{\text {th }}$ of February 2012

[8] de Souza e Silva, A. (2006) From Cyber to Hybrid: Mobile Technologies as Interfaces of Hybrid Spaces. [Online] Space and Culture Published by SAGE Publications, August 2006 [Accessed $1^{\text {st }}$ August 2012] http://sac.sagepub.com/content/9/3/261. 
[9] Dix, A., Gongora, L. (2011). Externalisation and Design. Paper at DESIRE'11, October 19-21, 2011, Eindhoven, the Netherlands. Proceedings of the Second Conference on Creativity and Innovation in Design.

[10] Dourish, P. (2006) Re-Space-ing and Place: in "Place" and "Space" Ten Years On Proceedings of CSCW 06, November 4-8 2006, Canada

[11] Gaver, W. Beaver, J. Benford, S. (2003) Ambiguity as a Resource for Design. Proceedings of CHI 2003, Ft. Lauderdale, Florida, ACM Press, New York, NY.

[12] Giannachi, G. Benford, S. (2008) Temporal Expansion in Blast Theory, Day of Figurines PAJ: A Journal of Performance and Art, PAJ 90 Published by MIT Press, September 2008 Volume 30 No 3 pp 60-69.

[13] Kim, H., Yoon, H., Choi, A., Baek, W., Lee, I., Kim, D., Woo, W. (2011). Data Markup

Representation for Mixed Reality Contents. International AR Standards Meeting-February 17192011.

[14] Koskinen, I., Zimmerman, J., Binder, T., Redstorm, J., Wensveen, S. (2011). Design Research Through Practice Form The Lab. Fielld. And Showroom. Published by Morgan Kaufmann.

[15] Kumar, V (2004). Innovation Planning Toolkit presented at FUTUREGROUND International Conference Melbourne, November 17-21, 2004

[16] Lawson, B. (2002). How Designers Think The Design Process Demystified. A completely revised third edition. First Published by Architectural Press 1980.

[17] Lee, Y., Oh, S., Woo, W. (2009). Ubiquitous Virtual Reality and Its Key Dimension. Proceedings of 2009 International Symposium on Ubiquitous Virtual Reality Conference, pp 5-8.

[18] Lifton, J., Laibowitz, M., Harry, D., Nan-Wei Gong., Mittal, M., Paradiso, J.A. (2009). Metaphor and Manifestation Cross-Reality with Ubiquitous Sensor/Actuator Networks. Pervasive Computing, IEEE Date: July-Sept. 2009 - Volume: 8 - Issue:3 pp $24-33$.

[19] Microsoft Research. (2011) Lightspace. [Online]. Available from: http://research.microsoft.com/enus/projects/lightspace/. [Accessed $6^{\text {th }}$ June, 2011]

[20] Milgram, P. Colquhoun Jr, H. 1999. A Taxonomy of Real and Virtual World Display. Integration

Y.Ohta and H.Tamura (Eds), Mixed reality; Merging real and virtual worlds pp5-28 New York; Springer
[21] Perry, M. Thomas, P. (1995) Externalising The Internal: Collaborative Design Through Dynamic Problem Visualisation Proceedings of $\mathrm{HCl}$ 1995

[22] Ramduny-Ellis, D., Dix, A.,Evans,M., Hare,J., Gill, S. (2010). Physicality in Design: an Exploration. The Design Journal Vol 13 Issue:1, 2010 pp 48-76

[23] Rettig M. (1994) Prototyping for tiny fingers communications of the ACM, 37 (4, April 1994) pp $21-27$

[24] Saffer, D. (2010). Designing for Interaction. (2nd edition). Creating Innovative Applications and Devices. Published by New Riders.

[25] Salter, Liora, and Alison Hearn. 1996. Outside the Lines: Issues in Interdisciplinary Research. Montreal: McGill-Queens University Press.

[26] Sanders, E. Stappers, P. (2008) Co Creation and the New Landscape of Design. Co Design, Volume 4 Issue 1 March pp 5- 18.

[27] Shedroff, N. Noessel, C. (2010) Make It So: Learning From Sci-fi Interfaces Workshop Proceedings of the $6^{\text {th }}$ International Conference on Intelligent Environments.

[28] Strober, H. M. (2011) Interdisciplinary Conversations Challenging Habits of Thought Stanford University Press, Stanford, California.

[29] Tassoul, M. (2009) _ Creative Facilitation $3^{\text {rd }}$ edition published VSDD Netherlands

[30] Ulrich, K. T and Eppinger, S. D. (2003) Product Design and Development. Published by McGraw-Hill. New York.

[31] Virtual Reality Application Centre lowa State University Mirage [online] Available at http://www.vrac.iastate.edu/facilities.php [Accessed on 15th June 2012]

[32] Weiser, M. (1991). The computer for the $21^{\text {st }}$ century. Scientific American, Communication Computers and Networks, Date September 1991 Special Issue pp 94 to 104.

[33] Willson, A.D., Izadi, S. Hilliges, O., GarciaMendoza., Kirk, D., (2008) Bringing Physics to the Surface Proceeding of UIST 08, October 19-22, 2008 California USA pp 67-76

\section{ACKNOWLEDGEMENTS}

This work was undertaken as part of the postdisciplinary HighWire programme at Lancaster University. HighWire is funded by the Digital Economy Programme: a Research Councils UK cross council initiative led by EPSRC and contributed to by AHRC, ESRC and MRC. 